Mise au point

\title{
Abcès hépatiques
}

\section{Liver abscess}

\author{
G. Rossi ${ }^{\text {a }}$, E. Lafont ${ }^{\mathrm{a}, *}$, L. Gasperini ${ }^{\mathrm{a}}$, S. Dokmak ${ }^{\mathrm{b}}$, M. Ronot ${ }^{\mathrm{c}}$, B. Rossi $^{\mathrm{a}}$, V. Zarrouk $^{\mathrm{a}}$, \\ B. Fantin ${ }^{\mathrm{a}, \mathrm{d}}$, A. Lefort ${ }^{\mathrm{a}, \mathrm{d}}$ \\ a Service de médecine interne, hôpital Beaujon, Assistance publique-Hôpitaux de Paris, 100, boulevard du Général-Leclerc, 92110 Clichy, France \\ b Service de chirurgie viscérale et digestive, hôpital Beaujon, Assistance publique-Hôpitaux de Paris, 100, boulevard du Général-Leclerc, 92110 Clichy, France \\ ' Service de radiologie, hôpital Beaujon, Assistance publique-Hôpitaux de Paris, 100, boulevard du Général-Leclerc, 92110 Clichy, France \\ d IAME, UMR1137, université Paris-Diderot, Sorbonne Paris Cité, 75890 Paris cedex 1, France
}

\section{N F O A R T I C L E}

\section{Historique de l'article :}

Disponible sur Internet le 14 septembre 2016

\section{Mots clés :}

Abcès hépatiques

Diagnostic

Étiologies

Traitement

\section{R É S U M É}

Les abcès hépatiques sont des affections rares et graves, dont l'incidence augmente ces dernières années en raison du vieillissement de la population, de l'avènement de la chirurgie pancréatique et hépatobiliaire en cancérologie et en transplantation, et de l'augmentation des facteurs d'immunodépression. Le tableau clinique étant très aspécifique, le diagnostic repose principalement sur l'imagerie et sur la ponction diagnostique. Le traitement est mal codifié, mais repose sur l'association d'une antibiothérapie prolongée, et d'un drainage.

(c) 2016 Société Nationale Française de Médecine Interne (SNFMI). Publié par Elsevier Masson SAS. Tous droits réservés.

\section{A B S T R A C T}

Liver abscess is a rare and severe infection. Incidence increases because of aging of population, advances in liver and biliary surgery including liver transplantation, and immunodeficiency factors. Diagnosis depends mainly on imaging and needle aspiration for microbiological identification. Treatment is based on antibiotics, percutaneous or surgical drainage, and control of the primary source.

๑ 2016 Société Nationale Française de Médecine Interne (SNFMI). Published by Elsevier Masson SAS.

All rights reserved.

\section{Introduction}

Les abcès hépatiques $(\mathrm{AH})$ sont des affections rares mais dont l'incidence augmente depuis une cinquantaine d'années, du fait du vieillissement de la population, de l'augmentation de l'incidence des cancers digestifs, et du développement des techniques chirurgicales, endoscopiques et radiologiques interventionnelles de pointe ces dernières années, dans le domaine de la pathologie abdominale [1]. L'incidence est variable selon l'origine géographique, semblant particulièrement élevée en Asie du Sud-Est [2].

Au début du vingtième siècle, la première cause d'AH était la pyléphlébite post-appendicite, et la mortalité attribuable aux $\mathrm{AH}$ était d'environ $80 \%$. Les moyens diagnostiques radiologiques, les techniques chirurgicales et radiologiques interventionnelles, et la

\footnotetext{
* Auteur correspondant.

Adresse e-mail : emmanuellafont5@gmail.com (E. Lafont).
}

maîtrise des antibiotiques s'étant considérablement améliorés au cours du vingtième siècle, la mortalité a diminué progressivement : de $65 \%$ en 1970, elle est passée à 24-30\% en 1990 puis à 14-20\% dans les années 2000.

Les hommes sont plus touchés ( 55 à $60 \%$ des cas). L'âge moyen a augmenté de 25-30 ans en 1970 à 50-60 ans dans les années 2000. Les facteurs de risque médicaux de survenue d'AH sont l'éthylisme chronique, une hépatopathie chronique surtout si associée à une anastomose bilio-digestive, une immunodépression ou un diabète [3-5].

\section{Physiopathologie}

La porte d'entrée est retrouvée dans deux tiers des cas. Quand aucune porte d'entrée n'est retrouvée, on parle d'abcès cryptogénique. La compréhension du mécanisme physiopathologique (Fig. 1) est essentielle à la prise en charge thérapeutique. 


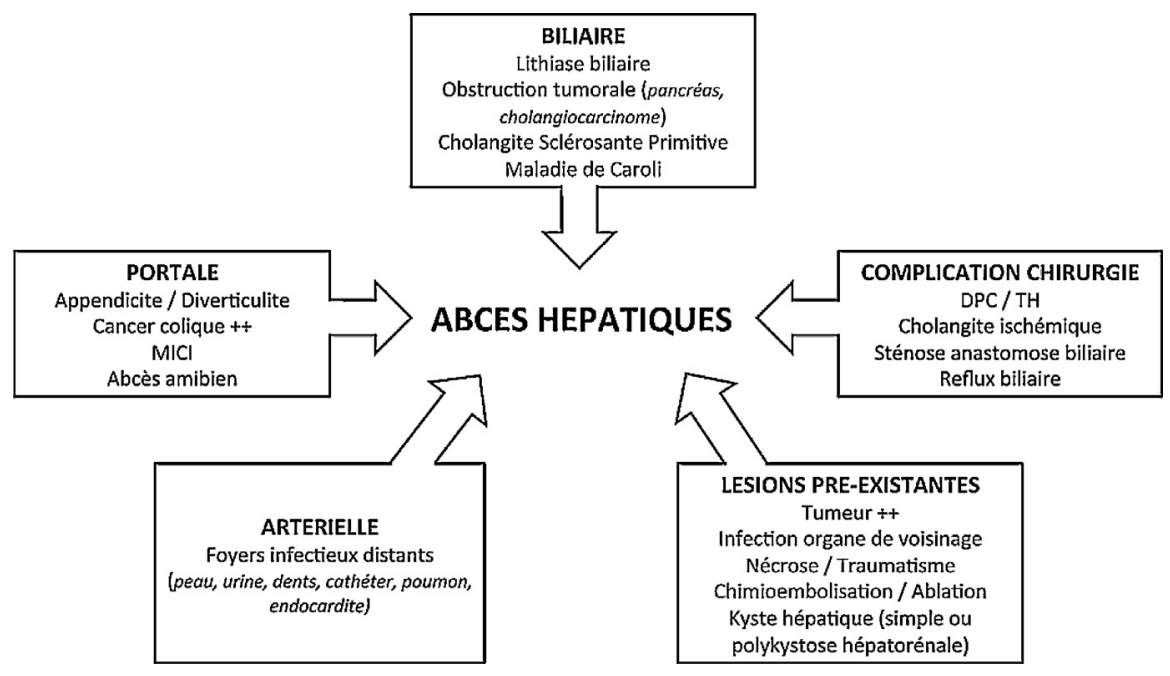

Fig. 1. Principales étiologies et mécanismes de formation des abcès hépatiques.

\subsection{Les abcès d'origine biliaire}

Ils représentant la première cause d'AH, sont souvent multiples et communiquent avec les voies biliaires [1-3]. Ils concernent volontiers le segment VI. On distingue les $\mathrm{AH}$ secondaires à une obstruction biliaire bénigne, qu'il s'agisse d'une cholécystite compliquée avec atteinte hépatique par contiguïté, ou d'une angiocholite sur lithiase biliaire, et les $\mathrm{AH}$ secondaires à une obstruction tumorale (ampullome, cancer de la tête du pancréas, cholangiocarcinome). Les maladies primitives des voies biliaires (cholangite sclérosante primitive, maladie de Caroli) sont des causes rares d'AH.

Les $\mathrm{AH}$ peuvent compliquer une chirurgie hépatobiliaire, par reflux biliaire dans l'anastomose bilio-digestive. Plusieurs cas d'AH à pyogènes dans les suites de gestes endoscopiques (par exemple au décours d'une cholangio-pancréatographie rétrograde par voie endoscopique : CPRE) ont été rapportés. Une obstruction de prothèse doit aussi être recherchée chez les patients porteurs d'une prothèse biliaire. La présence de bile dans le liquide drainé atteste de la présence d'une communication entre les voies biliaires et l'abcès, et doit donc faire rechercher une obstruction biliaire.

\subsection{Les abcès d'origine portale}

Ils représentent $10-20 \%$ des $\mathrm{AH}$, sont souvent polymicrobiens et localisés dans le foie droit. Ils proviennent de foyers infectieux digestifs surinfectés (appendicite, diverticulite, tumeurs coliques ou de lésions de maladies inflammatoires chroniques intestinales (MICI) par exemple) [4]. L'AH amibien est lié à la présence de micro-abcès dans la muqueuse rectale se drainant via les capillaires sanguins dans la circulation portale [4].

\subsection{Les abcès d'origine artérielle}

De mécanisme hématogène, ils sont plus rares, souvent monomicrobiens, et succèdent à une bactériémie ou à une fongémie d'origine extra-digestive [4].

\subsection{Les abcès liés à une surinfection de lésions préexistantes}

Les kystes hépatiques, les tumeurs hépatiques primitives ou secondaires, peuvent se surinfecter, notamment lorsque ces dernières ont fait l'objet d'une chimio-embolisation ou une d'ablation percutanée, particulièrement chez des patients ayant eu une sphinctérotomie [6,7]. En cas de polykystose hépatorénale, les kystes biliaires peuvent s'infecter plus fréquemment chez des patients ayant une insuffisance rénale dialysée. L'extension d'une infection de voisinage (cholécystite gangrenée, tumeur de l'angle colique droit surinfectée, ulcère perforé), peut donner des abcès par contiguiité.

Des abcès peuvent également succéder à un traumatisme hépatique. La sévérité du traumatisme et le recours à une artérioembolisation augmentent l'incidence de survenue d'AH. Le risque de développer un abcès est directement lié à l'extension de la nécrose du parenchyme (séquestre), du fait de phénomènes combinés d'ischémie biliaire et du parenchyme hépatique, favorisant le développement de germes anaérobies.

D'autres causes d'AH sont beaucoup plus rares : surinfection bactérienne de kyste hydatique, abcès consécutifs à la migration de corps étrangers tels qu'une arête de poisson ou un os de poulet, ces derniers se trouvant le plus souvent à la face inférieure du foie gauche ou du segment I, proches du cadre duodénal et de l'antre gastrique (Fig. 2) [4,8-10].

\subsection{Les abcès compliquant une chirurgie hépatique ou pancréatique}

Les $\mathrm{AH}$ peuvent compliquer une chirurgie pancréatique surtout après duodénopancréatectomie (DPC) selon trois mécanismes intriqués [1] :

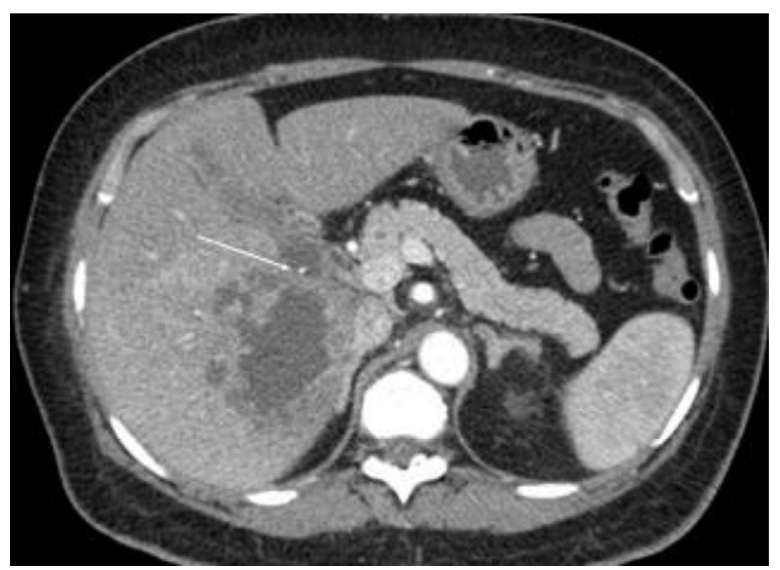

Fig. 2. Abcès hépatiques du secteur postérieur sur corps étranger (arête de poisson, flèche blanche). 
- des angiocholites en rapport avec un reflux au niveau de l'anse biliaire montée ou moins fréquemment une sténose de l'anastomose bilio-digestive ;

- une cholangite ischémique secondaire à une sténose organique de l'artère hépatique (par un traumatisme intra-opératoire ou par une embolisation postopératoire pour une hémorragie grave) ou fonctionnelle par une sténose du tronc cœliaque par un ligament arqué ou un athérome, qui n'a pas été diagnostiquée en préopératoire ;

- une cholangite ischémique localisée peut également s'observer en cas de sacrifice d'une artère hépatique aberrante (par exemple une artère hépatique droite naissant de l'artère mésentérique supérieure).

Ces $\mathrm{AH}$ à artère hépatique occlusive ont une mortalité très élevée (> 85 \%) en l'absence de revascularisation de l'artère hépatique $[11,12]$.

Après transplantation hépatique (TH), l'incidence d'AH est faible, entre 0,5 et $1 \%$, mais le risque de perte du greffon est élevé. Les facteurs de risque préopératoire de survenue sont essentiellement les greffons avec une ischémie chaude prolongée comme les donneurs à cœur arrêté. Les AH sur greffon hépatique peuvent être en rapport avec une sténose précoce organique ou fonctionnelle de l'artère hépatique (5-10\% des cas), avec une cholangite ischémique associée ou en rapport avec des complications biliaires (cholangiopathie) à artère hépatique perméable. Ces complications biliaires non anastomotiques avec une atteinte diffuse des voies biliaires ( $<5 \%$ des cas) ont une étiologie multifactorielle en rapport avec la durée de l'ischémie froide ou chaude, le liquide de conservation du greffon, immunologique ou virologique. Ces cholangiopathies diffuses avec artère hépatique perméable ou occluse nécessitent en général une retransplantation hépatique. Avec le développement de TH avec un split (partage d'un seul greffon hépatique pour deux receveurs, enfant-adulte ou pour deux adultes), un AH peut se développer au niveau du segment IV par sacrifice de son pédicule artériel lors du partage entre une lobe gauche et un foie droit, étendue partiellement au segment IV [13-15].

\section{Microbiologie}

\subsection{Les abcès hépatiques bactériens}

Les bactéries à croissance rapide représentent la cause la plus fréquente d'AH. Les bacilles à Gram négatif (BGN) sont impliqués dans 40-60\% des cas d'AH bactériens, particulièrement les entérobactéries (Escherichia coli, Klebsiella pneumoniae) et Pseudomonas aeruginosa. Les bactéries anaérobies représentent 35-45\% des bactéries en cause dans les $\mathrm{AH}$, en particulier Bacteroides sp. Les cocci à Gram positif (CGP), les plus fréquemment en cause sont Streptoccocus sp., Staphylococcus sp., Enterococcus sp. [16].

Dans 30 \% des cas, aucun germe n'est identifié. Les AH sont monomicrobiens dans 50-60\% des cas, et polymicrobiens dans 20-30\% des cas [17].

Les souches de K. pneumoniae hyper-virulentes sont responsables, depuis une vingtaine d'années, particulièrement en Asie du Sud-Est, d'une maladie émergente grave. L'incidence est maximale à Taiwan, où il s'agit du principal pathogène responsable d'AH, et où l'incidence atteint $80 \%$ dans certaines séries [18,19]. De plus en plus de cas sont rapportés aux États-Unis et en Europe [20], principalement chez des patients provenant de zone d'endémie.

Deux sérotypes de $K$. pneumoniae (K1 et K2) sont particulièrement virulents, et responsables d'un tableau associant une bactériémie, avec abcès multiples (abcès hépatiques le plus souvent monomicrobiens, endophtalmie, abcès cérébraux) (Fig. 3).

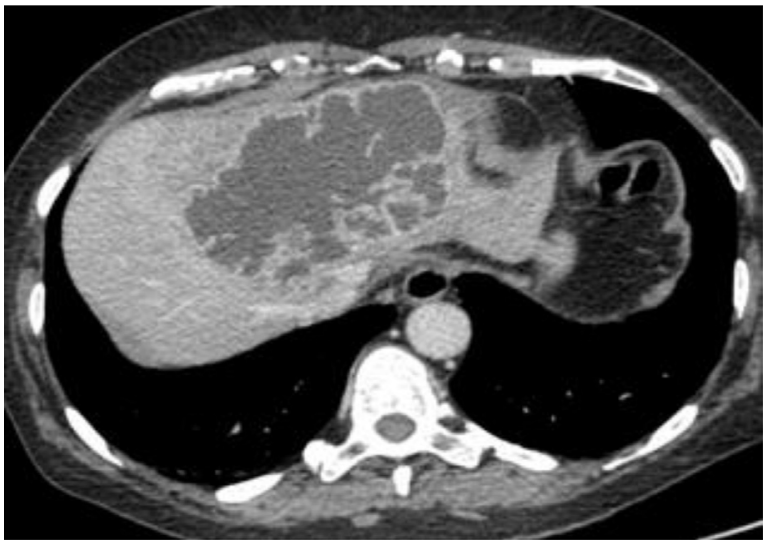

Fig. 3. Scanner au temps portal, abcès hépatique à Klebsiella pneumoniae.

\subsection{Les abcès hépatiques à bactéries à croissance lente ou non cultivables}

En cas d'AH sans germes pyogènes retrouvés, il faut rechercher des germes à croissance lente : mycobactéries, nocardioses, actinomycoses.

La survenue d'abcès à $M$. tuberculosis est surtout rapportée dans le cadre de tuberculoses disséminées, chez des patients immunodéprimés $[21,22]$. L'examen direct est rarement positif, mais la présence de granulomes peut orienter le diagnostic, et la culture positive le confirmer.

La présence de granulomes sur une ponction d'abcès peut également s'observer en cas d'infection à Brucella melitensis et Coxiella burnetii [23].

La nocardiose hépatique est rare, et concerne des patients immunodéprimés (VIH au stade SIDA, transplanté d'organe solide, hémopathie maligne, patient sous corticothérapie) [24]. L'AH à Actinomyces, localisation rare de la maladie, est le plus souvent d'origine portale, et fait suite à une brèche de la muqueuse digestive (appendicite, traumatisme colique). Il est nécessaire de réaliser des cultures prolongées sur milieux enrichis pour isoler cette bactérie [25].

\subsection{Les abcès hépatique amibiens}

La prévalence de l'amibiase tissulaire est devenue très faible en Europe $(0,7 \%)$, et concerne surtout les migrants. Le foie en est la localisation extra-intestinale la plus fréquente. La prévalence est forte en Afrique sub-saharienne, en Amérique Centrale, en Amérique du Sud et en Inde. Affectant dix fois plus les hommes que les femmes, l'âge médian se situe autour de 20-40 ans. Les kystes d'Entamoeba histolytica histolytica sont ingérés par l'intermédiaire d'eau contaminée. Les trophozoïtes forment des micro-abcès dans la muqueuse rectale. Les symptômes digestifs (syndrome dysentérique sans fièvre) passent souvent inaperçus. Ils atteignent ensuite le foie via le système porte. L'AH est le plus souvent unique, et localisé dans le foie droit.

La ponction de l'abcès ramène un pus stérile couleur chocolat. L'AH amibien peut se compliquer d'une rupture dans les organes de voisinage (plèvre, péritoine, péricarde), ce qui fait toute la gravité de la maladie. Le diagnostic, habituellement porté dans les 6 mois suivant le retour, repose sur la positivité de la sérologie [26].

\subsection{Les abcès hépatiques fongiques}

Les $\mathrm{AH}$ fongiques, dont l'incidence augmente, touchent l'immunodéprimé et sont dans la grande majorité des cas mixtes (fongiques et bactériens). Candida est le champignon le plus 


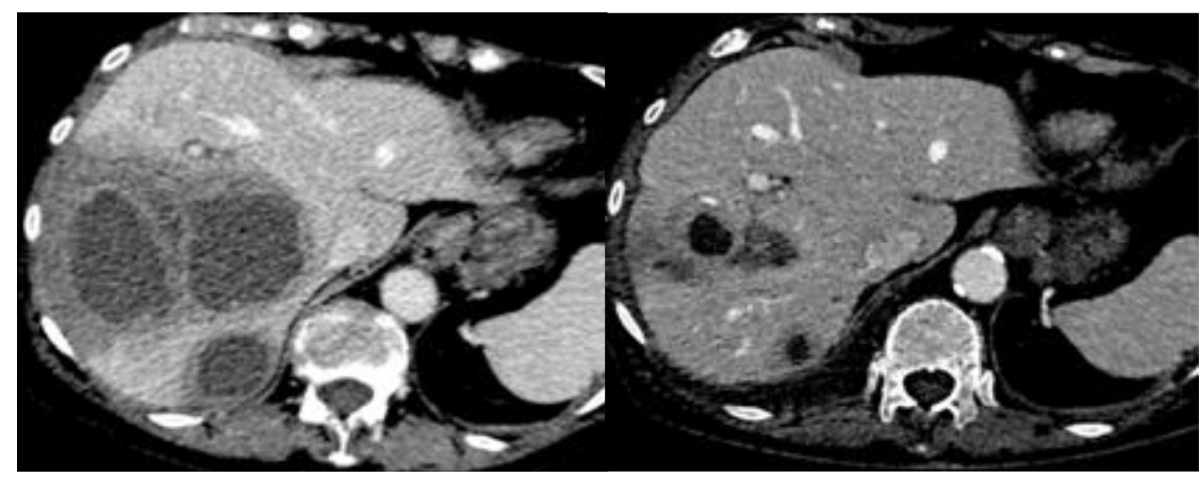

Fig. 4. Abcès hépatiques du foie droit avant traitement (à gauche), puis à 6 semaines de traitement (à droite).

fréquemment en cause dans les $\mathrm{AH}$, beaucoup plus rarement il existe des AH à Cryptococcus [27], Aspergillus [28], Mucorales [29].

La candidose hépatosplénique (CHS) constitue une forme particulière de candidose systémique chronique concernant les patients allogreffés de moelle osseuse et/ou atteints d'hémopathies malignes avec neutropénie profonde et prolongée. Les multiples abcès, disséminés dans le foie et la rate, apparaissent en sortie d'aplasie, du fait de phénomènes de restauration immunitaire. La recherche de Candida au sein des abcès ou des hémocultures est souvent négative [30].

\subsection{Les abcès hépatiques aseptiques}

Ces abcès, uniques ou multiples, de contenu stérile, sont localisés à la rate et au foie. Les patients présentent de la fièvre, un amaigrissement, des douleurs abdominales, des manifestations cutanées à type de pseudo-folliculite ou de dermatose neutrophilique. Il existe un syndrome inflammatoire biologique marqué.

Les abcès aseptiques sont souvent associés aux maladies inflammatoires chroniques intestinales ( $\mathrm{MICl}$, surtout la maladie de Crohn), mais leur survenue est indépendante de l'activité de la $\mathrm{MICI}$ associée. Des associations avec des polychondrites ou des gammapathies monoclonales de signification indéterminée ont été rapportées également. La grande cortico-sensibilité est un critère diagnostique important [31].

\section{Diagnostic}

\subsection{Clinique}

Le tableau clinique typique associe une fièvre (73-93\%), des frissons (43-80 \%) des douleurs de l'hypochondre droit (45-80\%), et une hépatomégalie douloureuse (30-50\%), un ictère (11-60\%), des vomissements (20-40\%), une perte de poids (14-50\%), une asthénie (25\%), une dyspnée (10-17\%), une toux (14\%) et une diarrhée (8-17\%).

Dans 15 à $19 \%$ des cas, l'AH se complique d'un choc septique. Plus rarement la présentation clinique peut être celle d'un abdomen chirurgical, d'une pneumopathie, ou d'une fièvre isolée [1-3].

\subsection{Biologie}

Les examens de routine montrent le plus souvent une hyperleucocytose (68\%), une élévation de la CRP, une hypoalbuminémie (70-96 \%), une élévation des phosphatases alcalines (66-71\%) et des gamma-glutamyl transférases (81\%), une cytolyse prédominant sur les alanine aminotransférases (53\%), et une hyperbilirubinémie (38-53\%) [1-3].

\subsection{Imagerie}

Les examens d'imagerie permettent de faire le diagnostic d'AH dans plus de $90 \%$ des cas, de rechercher une complication associée et d'orienter le diagnostic étiologique [32].

La radiographie thoracique peut montrer une ascension de la coupole diaphragmatique droite ou un épanchement pleural droit.

L'échographie hépatique $(\mathrm{EH})$ est souvent réalisée en première intention et montre à la phase pré-suppurative une lésion hyperéchogène avec des contours irréguliers, pouvant mimer une lésion tumorale, et à la phase suppurative une lésion hypoéchogène et régulière, parfois multiloculaire et entourée d'une fine capsule. Les micro-abcès ont un aspect nodulaire discrètement hypoéchogène. L'œdème péri-lésionnel aide à différencier l'AH d'un simple kyste hépatique. L'étude en Doppler doit rechercher une thrombose de la veine porte ou des veines hépatiques associée. L'EH permet aussi une étude des voies biliaires pour orienter le diagnostic étiologique.

Le scanner (Fig. 2 à 4) est plus sensible que l'EH et mène au diagnostic d'AH dans plus de $90 \%$ des cas. À la phase pré-suppurative, l'examen montre une lésion hypodense et hétérogène avec des contours irréguliers. Typiquement, la lésion présente un rehaussement périphérique au temps artériel et un aspect en cible au temps portal après injection. À la phase suppurative, l'aspect typique est dit " en cocarde ": lésion à centre hypodense avec rehaussement périphérique et œdème péri-lésionnel hypodense du parenchyme hépatique. La présence de gaz en son sein traduit la présence de bactéries anaérobies.

Les $\mathrm{AH}$ se compliquent, dans la moitié des cas, de thrombose des veines hépatiques ou portales segmentaires, localisées autour de l'abcès [33].

Les abcès à pyogènes et les abcès amibiens ne peuvent pas être distingués par l'imagerie seule. En échographie, les abcès amibiens apparaissent typiquement comme une lésion arrondie, unique, de grande taille, située dans le foie droit près de la capsule, hypoéchogène sans image de paroi associée. Au scanner, l'hypodensité est marquée avec une image de paroi fine et possiblement des cloisons au sein de l'abcès.

L'IRM hépatique n'est pas utilisée en routine en cas de suspicion d'AH, sauf en cas de suspicion de candidose hépatosplénique où ses performances diagnostiques sont supérieures à celles de l'échographie et du scanner. Les abcès à pyogènes ont des signaux d'intensité variable sur les images pondérées T1 et T2, avec un œdème péri-lésionnel en hypersignal en pondération T2. Les abcès amibiens apparaissent en hyposignal en pondération T1 et T2. Les abcès fongiques apparaissent en hyposignal en pondération T1, hypersignal en pondération T2. Ils se rehaussent après injection de chélates de gadolinium à la phase active de l'infection, et perdent leur hyposignal en pondération T1 au cours de la guérison.

La tomographie par émission de positons (TEP) est peu utilisée, sauf dans le diagnostic et le suivi d'une infection de kystes 
hépatiques dans le cadre de polykystose rénale autosomique dominante, où ses performances sont supérieures aux autres techniques d'imagerie [6]. Elle n'a pas été évaluée dans les autres contextes d'AH.

\subsection{Diagnostic microbiologique}

Les hémocultures sont négatives dans la moitié des cas d'AH. Elles ne permettent souvent pas d'isoler tous les microorganismes présents dans l'abcès [34].

La ponction d'abcès hépatique est l'examen de référence, et permet l'identification du (des) germe(s) dans près de $85 \%$ des cas. Elle permet en outre de drainer en partie l'abcès. Elle est le plus souvent réalisée sous guidage ultrasonore.

Dans le cas particulier de l'amibiase hépatique, le diagnostic repose essentiellement sur la sérologie sanguine, la ponction de l'abcès étant stérile (sauf en cas de surinfection bactérienne secondaire). Les techniques d'hémato-agglutination (HAI) et d'immunofluorescence indirecte (IFI) ont une sensibilité proche de $100 \%$, et l'utilisation conjointe des deux techniques permet de différencier une cicatrice sérologique d'une infection active. L'IFI se négative en 6 à 12 mois et l'HAI reste positive toute la vie [35].

\subsection{Recherche de la porte d'entrée}

La recherche de la porte d'entrée repose en grande partie sur l'imagerie. L'échographie hépatique est réalisée à la recherche d'une dilatation des voies biliaires, argument en faveur d'une obstruction biliaire, qu'elle soit d'origine lithiasique ou tumorale. Ses performances sont moins bonnes en cas de prothèse biliaire, car les voies biliaires sont souvent modérément dilatées en amont, même en cas de prothèse fonctionnelle. L'absence d'aérobilie dans le territoire en amont de la prothèse biliaire est en faveur de son obstruction. L'aggravation de la dilatation en comparaison avec des examens antérieurs est aussi un bon argument diagnostique. Devant des voies biliaires pathologiques, l'examen de référence est la cholangiographie par résonance magnétique (CP-IRM ou biliIRM). En périopératoire, l'absence d'une franche dilatation des voies biliaires ou d'un bilan hépatique peu perturbé ne doivent pas écarter formellement des complications infectieuses d'origine biliaire.

Le scanner abdomino-pelvien est souvent réalisé, pour éliminer un foyer infectieux digestif responsable d'AH d'origine portale. Dans les suites d'une chirurgie (comme la DPC ou la transplantation hépatique) ou d'un traumatisme, une sténose ou une plaie de l'artère hépatique doit être recherchée. En absence de porte d'entrée retrouvée, une coloscopie est indiquée. Enfin, en cas de dissémination hématogène, la recherche de la porte d'entrée sera adaptée au type de germe mis en évidence.

\section{Traitement}

Le traitement des AH associe traitement médical, éventuellement le drainage de l'abcès, ainsi que le traitement de la cause favorisante.

\subsection{Traitement médical}

Le traitement anti-infectieux doit être mis en route après avoir réalisé des hémocultures et si possible la ponction de l'abcès. Hormis les infections fongiques [26,36-38], il n'existe pas de consensus ni de recommandation concernant la prise en charge des abcès à pyogènes. Sur la base de notre expérience à l'hôpital Beaujon (Clichy), nous proposons habituellement un traitement parentéral pendant 10 à 14 jours, suivi d'un relais oral pour une durée totale de 3 à 4 semaines en cas d'abcès de petite taille ou drainé de manière satisfaisante, ou de 6 semaines en l'absence de drainage. La durée de traitement est conditionnée par la diminution du volume des lésions sur les examens d'imagerie de suivi. La Fig. 4 montre l'évolution d'un abcès bactérien du foie avant et après 6 semaines de traitement antibiotique.

En l'absence d'orientation, l'antibiothérapie probabiliste doit couvrir les germes digestifs les plus fréquemment responsables d'AH, c'est-à-dire les bacilles à Gram négatif aérobies, les anaérobies, et les streptocoques. En contexte communautaire, l'antibiothérapie probabiliste ne doit pas obligatoirement couvrir les entérocoques, et une association céphalosporine de $3^{\mathrm{e}}$ génération et métronidazole semble adaptée. En contexte nosocomial, une antibiothérapie à plus large spectre et active vis-à-vis des entérobactéries résistantes et des entérocoques est privilégiée ; celle-ci sera bien sûr discutée en fonction des antécédents infectieux du patient. En cas de choc septique, une association à un aminoside sera proposée. Toute antibiothérapie probabiliste fera l'objet d'une réévaluation et d'une adaptation à la documentation microbiologique.

Les schémas thérapeutiques habituellement utilisés sont indiqués dans les Tableaux 1 et 2.

\subsection{Aspiration et drainage de l'abcès}

L'antibiothérapie seule est la plupart du temps insuffisante pour le traitement des abcès de taille supérieure à $5 \mathrm{~cm}[39,40]$. Les techniques d'aspiration ou de drainage percutané ont alors un double avantage : permettre la documentation microbiologique et diminuer l'inoculum bactérien.

La ponction-aspiration consiste à aspirer le contenu de l'abcès sans mise en place d'un drain, contrairement à la ponction-drainage. Ces deux techniques ont des performances thérapeutiques comparable selon les études [41], cependant la récente

Tableau 1

Proposition de traitement des abcès hépatiques selon le résultat de l'hémoculture ou de l'examen direct du pus.

\begin{tabular}{|c|c|c|}
\hline Examen direct & Infection communautaire & Infection associée aux soins \\
\hline \multirow[t]{2}{*}{ Bacilles à Gram négatif } & Céfotaxime & Céfépime $100 \mathrm{mg} / \mathrm{kg} / \mathrm{j}$ \\
\hline & $100-150 \mathrm{mg} / \mathrm{kg} / \mathrm{j}$ & Si facteur de risque de BLSE : méropénème $100 \mathrm{mg} / \mathrm{kg} / \mathrm{j}$ \\
\hline \multirow[t]{2}{*}{ Cocci à Gram positif en chaînettes } & Amoxicilline & Vancomycine \\
\hline & $100-150 \mathrm{mg} / \mathrm{kg} / \mathrm{j}$ & $30-40 \mathrm{mg} / \mathrm{kg} / \mathrm{j}$ \\
\hline \multirow[t]{2}{*}{ Cocci à Gram positif en amas } & Oxacilline & Vancomycine \\
\hline & $100-200 \mathrm{mg} / \mathrm{kg} / \mathrm{j}$ & $30-40 \mathrm{mg} / \mathrm{kg} / \mathrm{j}$ \\
\hline \multirow[t]{2}{*}{ Flore polymorphe } & Métronidazole & Métronidazole \\
\hline & $500 \mathrm{mg} / 8 \mathrm{~h}$ & $500 \mathrm{mg} / 8 \mathrm{~h}$ \\
\hline \multirow[t]{2}{*}{ Levures ou filaments } & Amphotéricine B liposomale & Amphotéricine B liposomale \\
\hline & $5 \mathrm{mg} / \mathrm{kg} / \mathrm{j}$ & $5 \mathrm{mg} / \mathrm{kg} / \mathrm{j}$ \\
\hline \multirow[t]{3}{*}{ Examen direct négatif ou indisponible } & Céfotaxime + métronidazole & Céfépime + métronidazole \\
\hline & & ou \\
\hline & & Pipéracilline/tazobactam $4 \mathrm{~g} / 6 \mathrm{~h}$ \\
\hline
\end{tabular}

BGN : bacille à Gram négatif ; CGP : cocci à Gram positif ; BLSE : bêtalactamase à spectre étendu. 
Tableau 2

Proposition de traitement médical des abcès hépatiques selon le type de microorganismes.

\begin{tabular}{|c|c|c|}
\hline Microorganismes & Traitement intraveineux (IV) & Relais per os (PO) \\
\hline Enterobacteriaceae & $\begin{array}{l}\text { Amoxicilline } \\
\text { ou céfotaxime } \\
\text { ou céfépime } \\
\text { Si BLSE : méropénème }\end{array}$ & $\begin{array}{l}\text { Amoxicilline } 100 \mathrm{mg} / \mathrm{kg} / \mathrm{j} \text {, } \\
\text { ou amoxicilline } 100 \mathrm{mg} / \mathrm{kg} / \mathrm{j}+\text { clavulanate } 1200 \mathrm{mg} / \mathrm{j} \\
\text { ou lévofloxacine } 500 \mathrm{mg} / 12 \mathrm{~h} \mathrm{j} 1 \text { puis } 750 \mathrm{mg} / \mathrm{j}\end{array}$ \\
\hline Enterococcus & $\begin{array}{l}\text { Amoxicilline } \\
\text { Si E. faecium : vancomycine }\end{array}$ & $\begin{array}{l}\text { Amoxicilline } \\
\text { Si E. faecium : linézolide } 600 \mathrm{mg} / 12 \mathrm{~h}\end{array}$ \\
\hline Streptococcus & Amoxicilline & Amoxicilline \\
\hline Staphylococcus & $\begin{array}{l}\text { Oxacilline } \\
\text { Si SARM : vancomycine }\end{array}$ & $\begin{array}{l}\text { Lévofloxacine } 750 \mathrm{mg} / \mathrm{j} \\
\text { ou cotrimoxazole } 800 \mathrm{mg} \times 4 / \mathrm{j} \\
\text { ou clindamycine } 600 \mathrm{mg} \times 4 / \mathrm{j} \\
\text { (bithérapie PO pour Staphylococcus aureus) }\end{array}$ \\
\hline Pseudomonas aeruginosa & Ceftazidime $100 \mathrm{mg} / \mathrm{kg} / \mathrm{j} \pm$ amikacine & Ciprofloxacine $750 \mathrm{mg} \times 2 / \mathrm{j}$ \\
\hline Germes anaérobies & \multirow{2}{*}{\multicolumn{2}{|c|}{$\begin{array}{l}\text { Métronidazole } 500 \mathrm{mg} / 8 \mathrm{~h} \text { (même posologie IV et PO) } \\
\text { Métronidazole } 500 \mathrm{mg} \times 3 / \mathrm{j} \text {, durée } 2-3 \text { semaines, } \\
\text { puis tiliquinol-tibriquinol } 2 \mathrm{cp} / 12 \mathrm{~h} \text {, pendant } 10 \text { jours (l'amoebicide de contact permet d'éviter une ré-infestation) }\end{array}$}} \\
\hline Entamoeba histolytica histolytica & & \\
\hline Candida sp. & \multicolumn{2}{|c|}{$\begin{array}{l}\text { Amphotéricine B liposomale } 5 \mathrm{mg} / \mathrm{kg} / \mathrm{j} \text {, pendant } 4 \text { semaines, } \\
\text { puis fluconazole } 6 \mathrm{mg} / \mathrm{kg} / \mathrm{j} \text { PO, pendant } 3 \text { mois. (persistance de la fièvre sous amphotéricine B liposomale : } \\
\text { discuter prednisone } 1 \mathrm{mg} / \mathrm{kg} / \mathrm{j} 14 \mathrm{j} \text { ) }\end{array}$} \\
\hline Cryptococcus & \multicolumn{2}{|l|}{$\begin{array}{l}\text { Sans fongémie, ni atteinte neurologique centrale : flucon } \\
\text { Cryptococcose disséminé (situation la plus fréquente) : } \\
\text { amphotéricine B liposomale } 3 \text { à } 5 \mathrm{mg} / \mathrm{kg} / \mathrm{j} \text { + flucytosine } \\
100 \mathrm{mg} / \mathrm{kg} / \mathrm{j} \text { divisé en } 4 \text { fois, pendant } 2 \mathrm{semaines} \mathrm{par} \\
\text { voie intraveineuse, puis fluconazole } 400 \mathrm{mg} / \mathrm{j} \text { PO } \\
\text { pendant } 8 \text { semaines, puis fluconazole } 200 \mathrm{mg} / \mathrm{j} \text { PO } \\
\text { jusqu'à restauration immunitaire }\end{array}$} \\
\hline Aspergillus & \multicolumn{2}{|c|}{ Voriconazole $6 \mathrm{mg} / \mathrm{kg} / 12 \mathrm{~h}$ le premier jour, puis $4 \mathrm{mg} / \mathrm{kg} / 12 \mathrm{~h}$, durée totale 3 à 6 mois } \\
\hline
\end{tabular}

méta-analyse de Cai et al. [42] montre une supériorité du drainage. Les échecs du drainage percutané sont plus fréquents en cas d'AH difficile d'accès (lésion profonde, localisée au dôme hépatique), ou d'abcès multi-cloisonné [43]. Le drainage percutané peut se compliquer d'une rupture de l'abcès dans la cavité péritonéale, d'un hématome sous-capsulaire ou d'une contamination pleurale secondaire. Cependant ces complications sont plus rarement rencontrées qu'après un drainage chirurgical [44]. Les contre-indications du drainage percutané sont la présence d'une ascite, une pathologie abdominale nécessitant une laparotomie d'urgence ou un abcès rompu [44]. Le drainage percutané doit être déclive. Des rinçages quotidiens au sérum physiologique sont réalisés par le drain. Un contrôle échographique est réalisé à 48-72 heures de la pose pour évaluer la qualité du drainage et la bonne position de celui-ci. Lorsque le drain ne donne plus, et qu'un affaissement de l'AH est observé à l'échographie de contrôle, celui-ci peut être retiré.

Le drainage chirurgical, réalisable par voie laparoscopique ou cœlioscopique [45] permet une exploration complète du foie pour localiser les abcès (aide de l'échographie peropératoire), la mise en place de drains de gros calibres (adéquats pour l'évacuation de pus épais, de débris nécrotiques), la rupture des cloisons au sein de l'AH, et le lavage de la cavité péritonéale (en cas de rupture de l'abcès). Les indications actuelles sont l'échec du drainage percutané, les abcès multi-cloisonnés, les localisations difficiles d'accès, la rupture de l'abcès dans la cavité péritonéale. La taille de l'abcès n'est pas un critère de choix de la technique de drainage $[40,46]$.

En dernière intention, ou dans le cas de cause biliaire complexe, le chirurgien a recours à une segmentectomie [47].

\subsection{Traitement de la cause favorisante}

Pour les AH d'origine biliaire, un drainage des voies biliaires par voie radiologique percutanée, chirurgicale avec un drain transcystique ou sur un drain de Kehr, ou par voie endoscopique (prothèse biliaire) doit être réalisé en urgence. La pose de prothèse métallique, si elle est jugée nécessaire, le sera dans un second temps car elle ne doit pas être réalisée en contexte septique. Dans un deuxième temps, le traitement du facteur favorisant doit être réalisé (cholécystectomie, levée de l'obstacle sur les voies biliaires).

En cas de foyer infectieux digestif, la résection chirurgicale doit être réalisée sans délai (appendicectomie).

Pour les abcès survenant dans les suites d'une DPC ou d'une $\mathrm{TH}$, la prise en charge dépendra du mécanisme sous-jacent et du moment et du délai au diagnostic.

En ce qui concerne la sténose de l'artère hépatique avec une DPC :

- si le diagnostic est fait en préopératoire, un ligament arqué doit être cherché systématiquement afin d'être sectionné, une sténose significative par athérome à l'origine du tronc cœliaque ou de l'artère mésentérique supérieure doit être traitée en préopératoire par voie endovasculaire ou rarement chirurgicale avec la mise en place d'un stent et un antiagrégant plaquettaire ;

- si le diagnostic est fait en peropératoire, un ligament arqué doit être sectionné, un traumatisme de l'artère hépatique sera traité par un pontage ou plus rarement par une artérialisation de la veine porte et un athérome doit être traité par un pontage ou par voie endovasculaire à la sortie du bloc ;

- si le diagnostic est fait est postopératoire, tout doit être fait pour assurer le plus rapidement possible une vascularisation artérielle intrahépatique.

Une sténose ou reflux au niveau d'une anastomose biliodigestive peuvent être traitées par une dilatation percutanée avec ou sans prothèse (pathologie maligne), réfection chirurgicale (pathologie bénigne) ou une prolongation de l'anse montée. Rarement une hépatectomie segmentaire peut être indiquée.

Des cholangiopathies diffuses sur des greffons hépatiques sont traitées par un drainage biliaire endoscopique ou percutané mais en général des TH itératives sont nécessaires selon le contexte clinique [48]. Certaines formes rares mais unilatérales peuvent être traitées par des hépatectomies. Les abcès du segment IV sur split (partage d'un greffon) ont un pronostic favorable avec un traitement associant drainage percutané et biliaire endoscopique. 


\section{Conclusion}

Les abcès hépatiques sont des affections rares et graves, dont l'incidence est en augmentation. L'épidémiologie des abcès hépatiques a considérablement changé au cours de ces dernières décennies avec notamment l'augmentation de l'incidence de bactéries multi-résistantes et/ou hyper-virulentes, et l'apparition de pathogènes émergents chez les patients immunodéprimés.

Même si aucune recommandation thérapeutique n'est disponible, l'association d'un traitement anti-infectieux adéquat, d'un drainage pour les abcès volumineux et de la prise en charge des facteurs favorisant permet habituellement une évolution favorable.

\section{Déclaration de liens d'intérêts}

Les auteurs déclarent ne pas avoir de liens d'intérêts.

\section{Références}

[1] Huang CJ, Pitt HA, Lipsett PA, Osterman FA, Lillemoe KD, Cameron JL, et al. Pyogenic hepatic abscess. Changing trends over 42 years. Ann Surg 1996;223:600-7.

[2] Mischinger HJ, Hauser H, Rabl H, Quehenberger F, Werkgartner G, Rubin R, et al. Pyogenic liver abscess: studies of therapy and analysis of risk factors. World J Surg 1994; 18:852-7.

[3] Chiche L, Dargère $S$, Le Pennec $V$, Dufay $C$, Alkofer B. Abcès à pyogènes du foie. Diagnostic et prise en charge. Gastroenterol Clin Biol 2008;32:1077-91.

[4] Branum GD, Tyson GS, Branum MA, Meyers WC. Hepatic abscess. Changes in etiology, diagnosis, and management. Ann Surg 1990;212:655-62.

[5] Alvarez Pérez JA, González JJ, Baldonedo RF, Sanz L, Carreño G, Junco A, et al. Clinical course, treatment, and multivariate analysis of risk factors for pyogenic liver abscess. Am J Surg 2001;181:177-86

[6] Sallée M, Rafat C, Zahar J-R, Paulmier B, Grünfeld J-P, Knebelmann B, et al. Cyst infections in patients with autosomal dominant polycystic kidney disease. Clin J Am Soc Nephrol 2009;4:1183-9.

[7] Chen Y-C, Yeh T-S, Tseng J-H, Huang S-F, Lin D-Y. Hepatic hydatid cysts with superinfection in a non-endemic area in Taiwan. Am J Trop Med Hyg 2002;67:524-7.

[8] Glick WA, Simo KA, Swan RZ, Sindram D, Iannitti DA, Martinie JB. Pyogenic hepatic abscess secondary to endolumenal perforation of an ingested foreign body. J Gastrointest Surg 2012;16:885-7.

[9] Mohr AM, Lavery RF, Barone A, Bahramipour P, Magnotti LJ, Osband AJ, et al. Angiographic embolization for liver injuries: low mortality, high morbidity. J Trauma 2003:55:1077-81.

[10] de Sainte Marie B, Coze S, Grégoire E, Jean R, Durand JM, Chiche L. [Sudden epigastric pain]. Rev Med Interne 2015;36:634-5.

[11] Gaujoux S, Sauvanet A, Vullierme M-P, Cortes A, Dokmak S, Sibert A, et al. Ischemic complications after pancreaticoduodenectomy: incidence, prevention, and management. Ann Surg 2009;249:111-7.

[12] Yeo CJ, Cameron JL, Sohn TA, Lillemoe KD, Pitt HA, Talamini MA, et al. Six hundred fifty consecutive pancreaticoduodenectomies in the 1990s: pathology, complications, and outcomes. Ann Surg 1997;226:248-57.

[13] Nikeghbalian S, Salahi R, Salahi H, Bahador A, Kakaie F, Kazemi K, et al. Hepatic abscesses after liver transplant: 1997-2008. Exp Clin Transplant 2009; 7:256-60

[14] Cowie SE, Yoshida EM, Ryan AG, Chung SW, Buczkowski AK, Ho SG, et al. Hepatic abscesses after liver transplantation secondary to traumatic intrahepatic bile duct injuries in a cadaveric allograft donor. Transpl Int 2004;17:379-83.

[15] Tachopoulou OA, Vogt DP, Henderson JM, Baker M, Keys TF. Hepatic abscess after liver transplantation: 1990-2000. Transplantation 2003;75:79-83.

[16] Brook I, Frazier EH. Microbiology of liver and spleen abscesses. J Med Microbiol 1998;47:1075-80.

[17] Rahimian J, Wilson T, Oram V, Holzman RS. Pyogenic liver abscess: recent trends in etiology and mortality. Clin Infect Dis 2004;39:1654-9.

[18] Siu LK, Yeh K-M, Lin J-C, Fung C-P, Chang F-Y. Klebsiella pneumoniae liver abscess: a new invasive syndrome. Lancet Infect Dis 2012;12:881-7.

[19] Yu W-L, Ko W-C, Cheng K-C, Lee H-C, Ke D-S, Lee C-C, et al. Association between rmpA and magA genes and clinical syndromes caused by Klebsiella pneumoniae in Taiwan. Clin Infect Dis 2006;42:1351-8.

[20] Moore R, O'Shea D, Geoghegan T, Mallon PWG, Sheehan G. Communityacquired Klebsiella pneumoniae liver abscess: an emerging infection in Ireland and Europe. Infection 2013;41:681-6.
[21] Mourad MM, Liossis C, Algarni A, Kumar S, Bramhall SR. Primary hepatic tuberculosis in immunocompetent adults: a UK case series. Oxf Med Case Rep 2014;2014:148-50.

[22] Kaur P, Fishman JA, Misdraji J, Varma MC, Kotton CN. Disseminated Mycobacterium kansasii infection with hepatic abscesses in a renal transplant recipient. Transpl Infect Dis 2011;13:531-5.

[23] Ariza J, Pigrau C, Cañas C, Marrón A, Martínez F, Almirante B, et al. Current understanding and management of chronic hepatosplenic suppurative brucellosis. Clin Infect Dis 2001;32:1024-33.

[24] Cockerill FR, Edson RS, Roberts GD, Waldorf JC. Trimethoprim/ sulfamethoxazole-resistant Nocardia asteroides causing multiple hepatic abscesses. Successful treatment with ampicillin, amikacin, and limited computed tomography-guided needle aspiration. Am J Med 1984;77:558-60.

[25] Sharma M, Briski LE, Khatib R. Hepatic actinomycosis: an overview of salient features and outcome of therapy. Scand J Infect Dis 2002;34:386-91.

[26] Stanley SL. Amoebiasis. Lancet 2003;361:1025-34.

[27] Liu P-Y, Yang Y, Shi Z-Y. Cryptococcal liver abscess: a case report of successful treatment with amphotericin-B and literature review. Jpn J Infect Dis 2009;62:59-60.

[28] Chasan R, Patel G, Malone A, Finn M, Huprikar S. Primary hepatic aspergillosis following induction chemotherapy for acute leukemia. Transpl Infect Dis 2013;15:E201-5.

[29] Su H, Thompson GR, Cohen SH. Hepatic mucormycosis with abscess formation. Diagn Microbiol Infect Dis 2012;73:192-4.

[30] De Castro N, Mazoyer E, Porcher R, Raffoux E, Suarez F, Ribaud P, et al. Hepatosplenic candidiasis in the era of new antifungal drugs: a study in Paris 2000-2007. Clin Microbiol Infect 2012;18:E185-7.

[31] André MFJ, Piette J-C, Kémény J-L, Ninet J, Jego P, Delèvaux I, et al. Aseptic abscesses: a study of 30 patients with or without inflammatory bowel disease and review of the literature. Medicine (Baltimore) 2007;86:145-61.

[32] Mortelé KJ, Segatto E, Ros PR. The infected liver: radiologic-pathologic correlation. Radiographics 2004;24:937-55.

[33] Syed MA, Kim TK, Jang H-J. Portal and hepatic vein thrombosis in liver abscess: CT findings. Eur J Radiol 2007;61:513-9.

[34] Chemaly RF, Hall GS, Keys TF, Procop GW. Microbiology of liver abscesses and the predictive value of abscess gram stain and associated blood cultures. Diagn Microbiol Infect Dis 2003;46:245-8.

[35] Kurland JE, Brann OS. Pyogenic and amebic liver abscesses. Curr Gastroenterol Rep 2004;6:273-9.

[36] Pappas PG, Kauffman CA, Andes DR, Clancy CJ, Marr KA, Ostrosky-Zeichner L, et al. Executive summary: clinical practice guideline for the management of candidiasis: 2016 update by the Infectious Diseases Society of America. Clin Infect Dis 2016;62:409-17.

[37] Perfect JR, Dismukes WE, Dromer F, Goldman DL, Graybill JR, Hamill RJ, et al. Clinical practice guidelines for the management of cryptococcal disease: 2010 update by the Infectious Diseases Society of America. Clin Infect Dis 2010;50:291-322

[38] Walsh TJ, Anaissie EJ, Denning DW, Herbrecht R, Kontoyiannis DP, Marr KA, et al. Treatment of aspergillosis: clinical practice guidelines of the Infectious Diseases Society of America. Clin Infect Dis 2008;46:327-60.

[39] Bamberger DM. Outcome of medical treatment of bacterial abscesses without therapeutic drainage: review of cases reported in the literature. Clin Infect Dis 1996:23:592-603.

[40] Tan Y-M, Chung AY-F, Chow PK-H, Cheow P-C, Wong W-K, Ooi LL, et al. An appraisal of surgical and percutaneous drainage for pyogenic liver abscesses larger than $5 \mathrm{~cm}$. Ann Surg 2005;241:485-90.

[41] Yu S, Ho S, Lau W, Yeung D, Yuen E, Lee P. Treatment of pyogenic liver abscess: prospective randomized comparison of catheter drainage and needle aspiration. Hepatology 2004;39:932-8.

[42] Cai YL, Xiong XZ, Lu J, Cheng Y, Yang C, Lin YX, et al. Percutaneous needle aspiration versus catheter drainage in the management of liver abscess: a systematic review and meta-analysis. HPB 2015;17:195-201.

[43] Barakate MS, Stephen MS, Waugh RC, Gallagher PJ, Solomon MJ, Storey DW, et al. Pyogenic liver abscess: a review of 10 years' experience in management. Aust N Z J Surg 1999;69:205-9.

[44] Mezhir JJ, Fong Y, Jacks LM, Getrajdman GI, Brody LA, Covey AM, et al. Current management of pyogenic liver abscess: surgery is now second-line treatment. J Am Coll Surg 2010;210:975-83.

[45] Siu WT, Chan WC, Hou SM, Li MK. Laparoscopic management of ruptured pyogenic liver abscess. Surg Laparosc Endosc 1997;7:426-8.

[46] Wang W, Lee W-J, Wei P-L, Chen T-C, Huang M-T. Laparoscopic drainage of pyogenic liver abscesses. Surg Today 2004;34(4):323-5.

[47] Strong RW, Fawcett J, Lynch SV, Wall DR. Hepatectomy for pyogenic liver abscess. HPB 2003:5:86-90.

[48] Sheiner PA, Varma CV, Guarrera JV, Cooper J, Garatti M, Emre S, et al, Selective revascularization of hepatic artery thromboses after liver transplantation improves patient and graft survival. Transplantation 1997;64: 1295-9. 